\title{
Erratum to: Transportan 10 improves the anticancer activity of cisplatin
}

\author{
Izabela Rusiecka $^{1}$ - Jarosław Ruczyński ${ }^{2}$ Magdalena Alenowicz ${ }^{2}$ Piotr Rekowski ${ }^{2}$. \\ Ivan Kocić ${ }^{1}$
}

Published online: 10 March 2016

(C) Springer-Verlag Berlin Heidelberg 2016

\section{Erratum to: Naunyn-Schmiedeberg's Arch Pharmacol}

DOI 10.1007/s00210-016-1219-5

Unfortunately, it has been realized following online publication that an error has occurred in the author listing of the above article.

The author listing "Rusiecka Izabela, Ruczyński Jarosław, Alenowicz Magdalena, Rekowski Piotr, Kocić Ivan” should read correctly "Izabela Rusiecka, Jarosław Ruczyński, Magdalena Alenowicz, Piotr Rekowski, Ivan Kocić". Moreover, the name of the corresponding author should read "Ivan Kocić" in place of "Kocić Ivan". We apologize for any confusion this error may have caused.

The online version of the original article can be found at http://dx.doi.org/ 10.1007/s00210-016-1219-5.

Ivan Kocić

ikocic@gumed.edu.pl

1 Department of Pharmacology, Medical University of Gdańsk, Gdańsk, Poland

2 Department of Chemistry, University of Gdańsk, Gdańsk, Poland 